\title{
Hypogastric Artery Management During Open and Endovascular Aortoiliac Repair
}

\author{
Aaron Fargion, Carlo Pratesi, Fabrizio Masciello, \\ Walter Dorigo, and Giovanni Pratesi
}

\subsection{Demographic, Pathogenesis, and Natural History}

While iliac artery aneurysms (IAAs) are frequently associated with abdominal aortic aneurysms (AAAs) [1, 2], isolated IAAs are rare, with a frequency between $0.5 \%$ and $2 \%$ of all arterial aneurysms [3] and an incidence among general population of about $0.03 \%$ [4].

IAAs are defined as an increase in iliac artery diameter of at least $100 \%$ [2]; and considering that a normal common iliac artery has an approximate diameter of $1.2 \mathrm{~cm}, 2.5 \mathrm{~cm}$ is considered the threshold limit for the definition of IAAs [5]. As far as general demographic data are concerned, IAAs are more frequent in men, with a 5:1 ratio with women, and in patients aged 70 or older [2]. More than $70 \%$ of IAAs involve the common iliac artery (CIA), between 20 and $25 \%$ involve the hypogastric artery (HA), and isolated external iliac artery (EIA) aneurysms are quite rare, probably due to its different embryogenesis [6]. Frequently an aneurysmatic lesion is detected in more than one iliac artery, and it is bilateral in almost one third of the patients (Fig. 8.1).

\footnotetext{
A. Fargion · C. Pratesi $(\triangle) \cdot$ F. Masciello $\cdot$ W. Dorigo Department of Vascular Surgery, University of Florence, Florence, Italy e-mail: carlo.pratesi@unifi.it

G. Pratesi

Department of Vascular Surgery, University of Rome Tor Vergata, Rome, Italy
}

It has been suggested that IAAs share the same multifactorial pathogenetic pathway promoting the development of AAAs. More than $90 \%$ of the lesions has an atherosclerotic cause; among other causes, collagenopathies (Marfan's syndrome, Ehlers-Danlos' syndrome) and inflammatory arterial diseases (Takayasu's disease, Behçet's disease, and polyarteritis nodosa) have to be considered. Infective aneurysms are quite rare and often anecdotally reported.

As AAAs, their natural history contemplates a constant lesion growth, progressively increasing risk of aneurysmal rupture. In the largest published study reporting the fate of untreated IAAs, Santilli

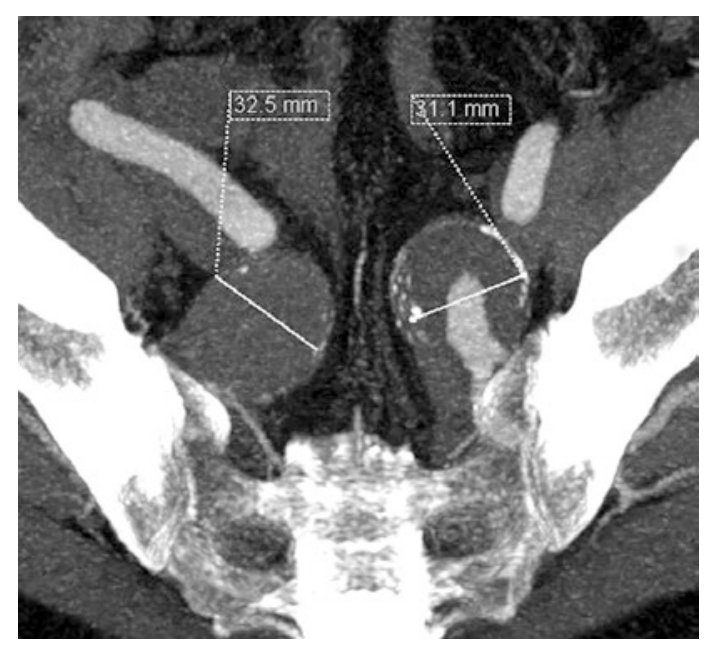

Fig. 8.1 MIP reconstruction of bilateral internal iliac aneurysms 
et al. [7] followed over 300 people with iliac aneurysms of $<3 \mathrm{~cm}$ in diameter over a 4-year period and found a slow average expansion rate of $1 \mathrm{~mm} /$ year. In this study growth rate accelerated to $2.6 \mathrm{~mm} /$ year when iliac diameter exceeded $3 \mathrm{~cm}$. Furthermore, expansion rates of isolated iliac aneurysms were similar regardless of laterality, presentation site, and association with an AAA. Differently, other studies suggest for IIA expansion comparable to AAAs [8]. Unfortunately, despite general growth rates, current literature on rupture risk by aneurysmal size is scarce. Currently available information is based on a series of large, symptomatic iliac aneurysms or autoptic findings on the diameters of ruptured IAAs (mean diameter $\sim 5.5 \mathrm{~cm}$ ) $[1,2]$. Considering the rupture rate of untreated IAAs with a maximum diameter of $>3 \mathrm{~cm}$ ranging between 14 and $70 \%$ and that perioperative risk in elective and urgent conditions varies from 3-5\% [3] to 30\%, current guidelines recommend this threshold as an indication for surgical repair [9, 10]. However, a recent survey involving more than 500 members of the Vascular Societies of Great Britain and Ireland showed that most physicians would wait until an IAA reached $4 \mathrm{~cm}$ in diameter before considering intervention [11]. Obviously, in the presence of symptoms and/ or of lesions with a $5.5 \mathrm{~cm}$ diameter, a prompt or urgent intervention is required.

\subsection{Clinical and Instrumental Diagnosis}

\subsubsection{Clinical Diagnosis}

IAAs are usually asymptomatic, and diagnosis is made by the patient, who reports a pulsatile mass in the abdomen, and by the physician during a clinical examination or during instrumental examinations performed for unrelated causes. The presence of an IAA must be suspected in the evidence of a pulsatile mass in the lower abdominal regions; in patients with distal CIAAs or HAs, such a finding cannot be appreciated but sometimes can be found during rectal examination.

However, differently from what usually happens in patients with AAAs, such lesions are more prone to cause compression to adjacent structures, which can be found in up to $30 \%$ of cases. The compression of the ureter can lead to hydronephrosis associated with urinary infections or hematuria, whereas the rectal compression can cause pain and tenesmus. Pelvic nerves can also be compressed, causing hypoesthesia of the lower limb, while radicular compression can cause sciatic pain. When rupture occurs, it is usually contained in the retroperitoneal space, causing typical lumbar and flank pain, often referred also to the groin and to the gluteal region. Contained rupture can cause long-lasting symptoms (days or weeks) due to the inflammation and distension of the retroperitoneum. Usually, the classical triad of lumbar pain, hypotension, and pulsatile mass in the iliac region is a strong index of suspicion for a contained rupture of an IAA. Open rupture with massive bleeding is rare at first presentation but represents the final event of an undiagnosed contained rupture. Rarely a contained rupture can occur in adjacent structures, such as iliac veins, causing acute congestive right heart failure, or rectum and ureter, causing acute or chronic bleeding.

Thrombosis or embolism of the aneurysm may cause other possible complications. Aneurysmal thrombosis can cause acute limb ischemia or, more frequently, gluteal or thigh claudication with impotence. Peripheral embolism is usually referred to microembolic events, causing a "blue toe syndrome," but patients may also present an acute/subacute limb ischemia due to macro-embolization.

\subsubsection{Instrumental Diagnosis}

The first-level diagnostic tool is the duplex ultrasound (DUS), which can identify the presence of the IAA with high accuracy and reproducibility and low costs [12]. Even with all the limits related to the patient conformation [13], DUS is reliable in detecting these aneurysms and assessing their diameters. Moreover, it can help in the diagnosis of impending rupture, if modifications of intraluminal thrombus, sudden enlargement, or presence of periarterial fluid or thickening are found. Angio-computed tomography $(\mathrm{CT})$ scan, even if more expensive and not 
applicable to all patients, is the gold standard in the evaluation of the morphological presentation of the lesion and in the evaluation of its proximal and distal extension. Its use is especially suggested in patients addressed to surgical repair, particularly when an endovascular procedure has to be planned, thanks to the possibility of 3D reconstructions with dedicated software. It is also mandatory in stable patients with suspected rupture, to assess endovascular feasibility and to accurately depict the status of other abdominal vessels. Angio-magnetic resonance (MR) scan, even if it provides similar accuracy to angio-CT in anatomical evaluation of IAAs, is more expensive, and high-resolution machines $(>1.5 \mathrm{~T})$ are usually less accessible. It may be useful in patients with contraindications to iodine contrast medium, and it represents an alternative to angio-CT scan in selected patients but does not allow to properly assess vessel calcifications. Digital subtraction angiography (DSA) is no longer used as a diagnostic tool due to its invasiveness and to the related local and general risks.

\subsubsection{Prevention and Medical Treatment}

Due to the lack of knowledge concerning the pathophysiology of IAA and of aneurysmal disease in general, a real prevention strategy still does not exist. Congenital factors associated with the development of IAAs are not yet possible to detect and correct. For this reason, strict control of commonly acquired risk factors for atherosclerosis is crucial in controlling lesion growth, rather than their appearance, so as to avoid the end-stage rupture. Smoking has been shown to be associated with an increased aneurysmal growth; for this reason, smoking cessation is mandatory. Several drug treatments, used to control cardiovascular risk factors, have been proposed to reduce aneurysmal growth rate during surveillance; however, no strategy has been demonstrated to be beneficial [14]. ACE inhibitors and statins seem to determine a slowing increase of AAAs, and this could also be suggested in patients with IAAs [15]. For other drugs ( $\beta$-blockers, doxycycline, roxithromycin), the benefit is uncertain, and their routine use is not recommended. Future developments could come from micromolecular techniques (proteomics, genomics, microRNA), possibly allowing to create drugs selective for the arterial wall, preventing its susceptibility to aneurysmal degeneration.

Since no formal recommendations for the surveillance of iliac aneurysms exist, most clinicians have adapted schedules used for small AAAs, monitoring them more frequently with increasing size. Even if the documented slow expansion rate of iliac aneurysms $<3 \mathrm{~cm}$ in diameter would support a policy of annual monitoring [16], many opt to multiply controls when they reached $2.6-3 \mathrm{~cm}$. New research into surveillance intervals for AAAs, however, has suggested that current schedules remain overcautious and could be significantly longer without increasing rupture rate [17].

\subsubsection{Surgical Treatment}

The aim of invasive treatment of IAAs is to surgically replace affected iliac segments or to exclude lesions from the blood flow reducing arterial wall stress, to prevent further dilatation and rupture.

As previously reported [7, 9, 10, 18], current guidelines suggest treating asymptomatic lesions with a diameter exceeding $3 \mathrm{~cm}$ and symptomatic ones independently from the diameter. Smaller and asymptomatic lesions should be followed up with DUS evaluation following the schedule previously described. Current treatment options include open surgery and endovascular repair. The choice for one of the two options derives from the careful evaluation of patients' general operative risk and lesion morphology [19].

\subsubsection{Open Surgery}

Conventional open surgical repair represented the only option for patients with AAAs and IAAs for nearly 50 years, and it is still widely used in clinical practice. It consists in aneurysmal resection with graft substitution (aneurysmectomy and graft inter- 
position). The intervention is usually performed under general anesthesia, with a urinary catheter, a nasogastric tube, a central venous line, and an arterial line preliminary placed. In recent years, a fasttrack strategy has increasingly been used, to reduce intra- and postoperative patient stress by minimizing the gastrointestinal injuries, using locoregional anesthesiological techniques, and promoting an early postoperative nutrition [20].

Access to the iliac vessels is achieved with two different approaches: median transperitoneal and retroperitoneal. The former is commonly used in patients with bilateral lesions or with a concomitant AAA, while the latter is preferred in patients with unilateral iliac involvement. During aneurysmal dissection (Fig. 8.2), attention must be paid to avoid injuries to adjacent structures, mainly iliac veins, which lie in a deeper plane and can cause severe bleeding, ureters, which lie above and laterally the iliac axis, and nerves (pelvic plexus).

Before the proximal and distal vessels are clamped, systemic heparinization is performed, usually at the dose of $30 \mathrm{IU} / \mathrm{kg}$; longitudinal incision of the sac is then performed, and the intraluminal thrombus is evacuated. The following step is the reconstitution of the arterial continuity.

In the presence of saccular aneurysms or limited blisters or blebs of the arterial wall, aneurysmal resection with primary closure of the arteriotomy can be safely performed. In most cases, however, a prosthetic graft interposition is required, whose extension and morphology depend on the aneurysm extension and on number of involved vessels.

In patients with IAA of the CIA, if adequate proximal and distal neck are available, a tube graft can be used to replace the affected artery in an "endto-end" fashion; if the iliac bifurcation is involved, the ostium of the HA should be included in the anastomosis cuff. If this is not possible, the distal anastomosis should be performed on the EIA or the HA and the other vessel reimplanted on the graft.

A similar strategy should be used also in case of concomitant aneurysms of the CIA and HA or isolated lesions of the HA, especially when bilateral. Even if several authors suggested in the past years that bilateral ligation of hypogastric arteries was relatively safe and advocated selective IMA reimplantation to prevent pelvic ischemic

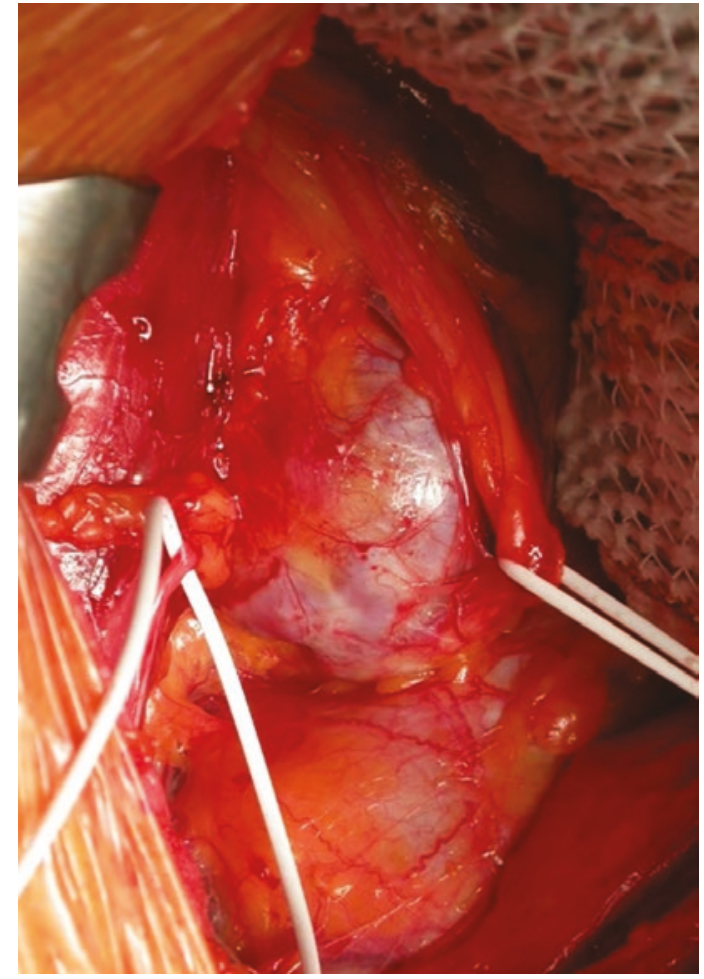

Fig. 8.2 Laparotomic isolation of an isolated iliac aneurysm

complication (stump pressure cutoff value $<0.6$ compared to radial pressure) [21], the vast majority of the surgeons agree to preserve at least one side, paying particular attention to the IIA status (extent of dilation and patency of outflow branches). If the aneurysm is limited to the proximal segment of the artery, a direct revascularization with a short graft starting either from native CIA or from a tube graft between CIA and EIA (" $\mathrm{T}$ " fashion reconstruction) is the ideal strategy; if the aneurysm involves the anterior and posterior branches of the vessel, an aneurysmorrhaphy with inside ligature of the branches is suggested. Unfortunately, depending on the size of the aneurysmal sac, surgical dissection down into the pelvis to isolate iliac branches may be extremely difficult and exposes the patient to an increased risk of bleeding and damage of adjacent structures. Recently a hybrid device made of an expanded PTFE prosthesis with a nitinol-reinforced self-expanding section at one end has been used to address this issue. This device, originally 
developed for hemodialysis accesses, was already implanted during complex aortic procedures for arch or visceral debranching allowing a sutureless revascularization of target vessels and decreasing overall clamping time [22]. The nitinol end is inserted on a $0.035^{\prime \prime}$ guidewire inside the target artery directly through the ostium (direct cannulation) or with a small incision on the side wall avoiding extensive surgical dissection. After the self-expanding portion is released distally and post-dilated, it is ensured with three 5-0 or 6-0 polypropylene stitches to avoid possible migrations. The standard PTFE side is then anastomosed end to side on a graft or native artery. This technique was originally proposed to treat complex thoracoabdominal aneurysms using standard self-expanding covered stents as bridges for visceral arteries to be sewn to the aortic tube graft or native iliacs, as in hybrid procedures, and was defined as "VORTEC" technique [23-25]. Finally, in patients with unilateral or bilateral iliac aneurysms associated with AAA, the surgical strategy usually adopted consists in a bifurcated graft distally brought to the EIAs or HAs with concomitant reimplantation or bypass of the remaining vessels (Fig. 8.3).

Open surgical treatment of IAAs in an elective setting is, nowadays, a safe procedure. Due to improvements in anesthesiological techniques and postoperative medical protocols, perioperative mortality rates have progressively decreased over the past years, and in most recent series, it does not exceed 2\% [26]. As expected, in urgent conditions, mortality rises to $7-10 \%$. The rate of nonfatal perioperative complications is however not negligible, ranging from 5\% [2] up to $15 \%$ [26]. Complications could be systemic or local. Among the former, the most common are cardiac (acute myocardial infarction, congestive heart failure), respiratory (pneumonia, acute respiratory insufficiency), and renal. Among local complications, the most frequent is the early postoperative bleeding, sometimes requiring surgical revision. Thrombosis of the graft leading to limb ischemia is rare but requires surgical revascularization; the most feared complications are related to pelvic and intestinal ischemia, due to both hemodynamic (prolonged hypotension,

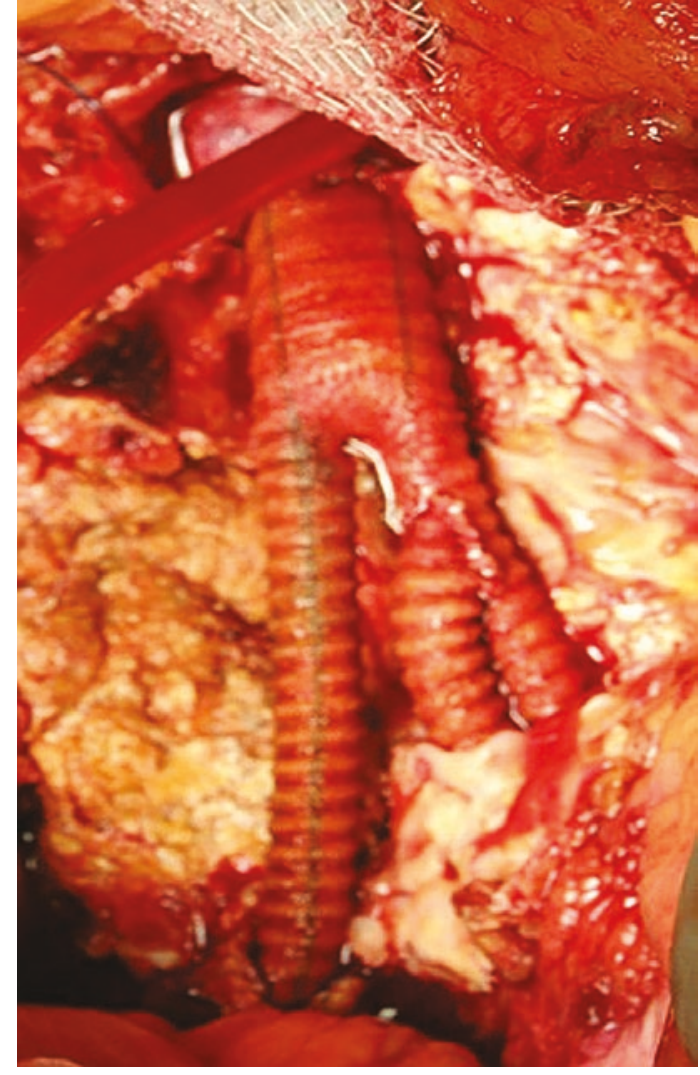

Fig. 8.3 Aorto-bi-iliac graft and end-to-side bypass to the internal iliac

acute bleeding) and surgical factors (lack of preservation of hypogastric flow, hypogastric diffuse microembolization). Other minor complications include wound infection, reported in up to $10 \%$ of cases, and wound dehiscence, for which the reported frequency is lower than $1 \%$.

The risk of late intervention-related complications is low, rarely exceeding $5 \%$ at 5 years, and the long-term survival of the operated patients, once the perioperative risk is overcome, is similar to a matched population for age and sex, without IAAs [27]. Among graft-related complications, anastomotic aneurysms (dilation of the arterial wall sutured to the graft), graft thrombosis, and infection have to be cited. More frequently, patients may develop new aneurysms proximally or distally to the intervention site during followup. Particularly, the most studied issue is the possible aneurysmatic evolution of the abdominal 
aorta years after treatment of an isolated IAA. Regarding this, there is no clear evidence in the literature concerning replacement of an ectatic abdominal aorta in patients with IAAs (maximum diameter lower than $3 \mathrm{~cm}$ ). Some authors suggest that the risk of AAA development in patients with mild dilatation of the aorta is substantial, and for this reason, it should be treated along with the IAA [28]. More recent studies showed, on the contrary, that a $5 \mathrm{~mm}$ increase in the diameter of the abdominal aorta after 5 years occurs only in $15 \%$ of patients operated on for IAA, suggesting the possibility of effectively following those patients up over time, leaving the ectatic abdominal aorta at the time of the first operation $[29,30]$.

\subsubsection{Endovascular Repair}

Endovascular aneurysm repair of abdominal aortic aneurysms (EVAR), conceived and realized by $\mathrm{N}$. Volodos in the Soviet Union and by J. Parodi in Argentina and the United States [31], represented a real revolution in the management of aortoiliac aneurysmal disease. Initially, it was reserved for elderly patients in poor general condition at high risk for open surgery, but nowadays it is increasingly used also in patients at intermediate risk, providing excellent results, mainly in the perioperative period. The use of endovascular techniques in the iliac vessels took its origin as a consequence of the frequent involvement of the iliac arteries in patients with AAA, and it was later applied to patients with isolated IAAs, considering that anatomical and technical issues are quite similar in those subgroups of patients. In fact, the involvement of iliac arteries significantly increases the complexity of the endovascular repair, negatively affecting early outcomes and late graft-related complications [32].

The main issue during endovascular management of IAAs is related to the lack of adequate distal neck in the CIA resulting in the need to find a suitable landing zone in the EIA. In the first experience, the only possible solution was to land with the endograft iliac limb down into the EIA and overstenting the HA ostium. This technique usually required a concomitant embolization of the HA, to prevent the risk of type II endoleak development. The embolization can be performed before or during endograft implantation, placing coils or plugs in the HA main trunk or in division branches (Fig. 8.4).

This procedure is associated with several complications addressed to the development of pelvic ischemia. In several different series, up to $30 \%$ of the patients undergoing HA embolization reported a combination of bowel, spinal
Fig. 8.4 (a) IIA embolization and (b) overstenting of the IIA ostium
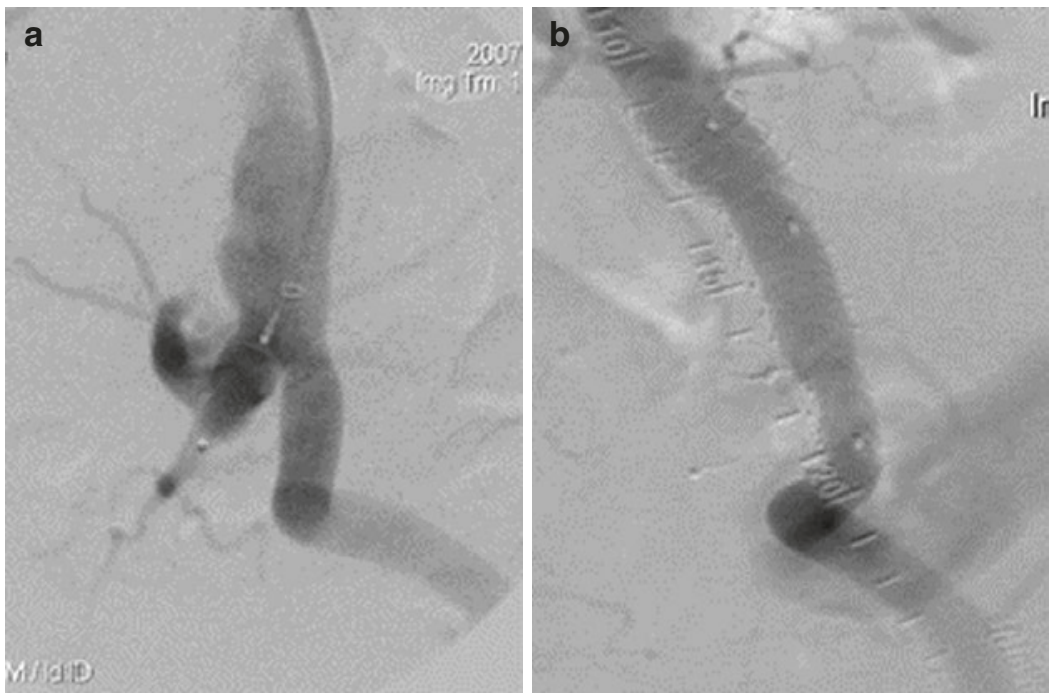
cord, or nerve ischemia, sexual impotence, buttock or thigh claudication, and buttock necrosis [33-37]. A systematic review [33] of 18 studies, including 643 patients with unilateral or bilateral HA embolization, reported $31 \%$ vs $35 \%$ of buttock claudication and $17 \%$ vs $24 \%$ of impotence, respectively. There are few data in the literature concerning risk factors for the developing symptoms of pelvic ischemia when an HA occlusion is performed; among them, presence of diabetes, multilevel occlusive disease, and differences in patients' age have been largely examined.

Buttock claudication has a higher presentation rate in younger patients with an active lifestyle and in elderly patients with reduced inflow due to cardiac disease. Moreover, in patients with concomitant occlusive disease of the contralateral HA and/or of the ipsilateral common femoral artery, symptoms are frequent due to lack of collateral flow to the embolized vessel [38]. In these subgroups of patients, antegrade flow in the HA should be preserved to avoid pelvic ischemia. This issue can be achieved with different techniques during open surgical repair (see description above) or hybrid procedures [39] (Fig. 8.5a). Several possible endovascular options have been used: the bell-bottom technique, the multilayer stent technology, the chimney or sandwich technique, the iliac branch device, and, more recently, the endovascular aneurysm sealing.

The bell-bottom technique, proposed by Karch et al. [40] in 2001, consists of an off-label use of an aortic proximal cuff to achieve sealing in ectatic CIAs with diameter between 14 and $18 \mathrm{~mm}$. Although designed for the proximal aortic neck, the extension cuffs were implanted into the distal portion of the iliac limb. The result was a flared iliac limb in a bell-bottom configuration, which achieved adequate hemostatic seal in the enlarged or aneurysmal iliac segments (Fig. 8.6). For many years, large-diameter iliac limbs have been commercially available on the European market. Even if long-term results are favorable [41], flared limb size limits such technique to iliacs of up to $24 \mathrm{~mm}$ in diameter in the seal zone.

A multilayer stent is made of a threedimensional matrix of nitinol wires derived from neuroradiological concept of flow diverter stents. The theory behind the function of this technology is the action on blood flow: reducing turbulence inside the aneurysmal sac and maintaining laminar flow into the main artery and its collaterals. Even if the aneurysmal sac is not immediately excluded from the blood flow after its placement, the progressive reduction of flow velocity potentially favors intra-sac thrombus formation with aneurysmal exclusion, while main collaterals tend to remain patent (Fig. 8.5b). While there are several published series
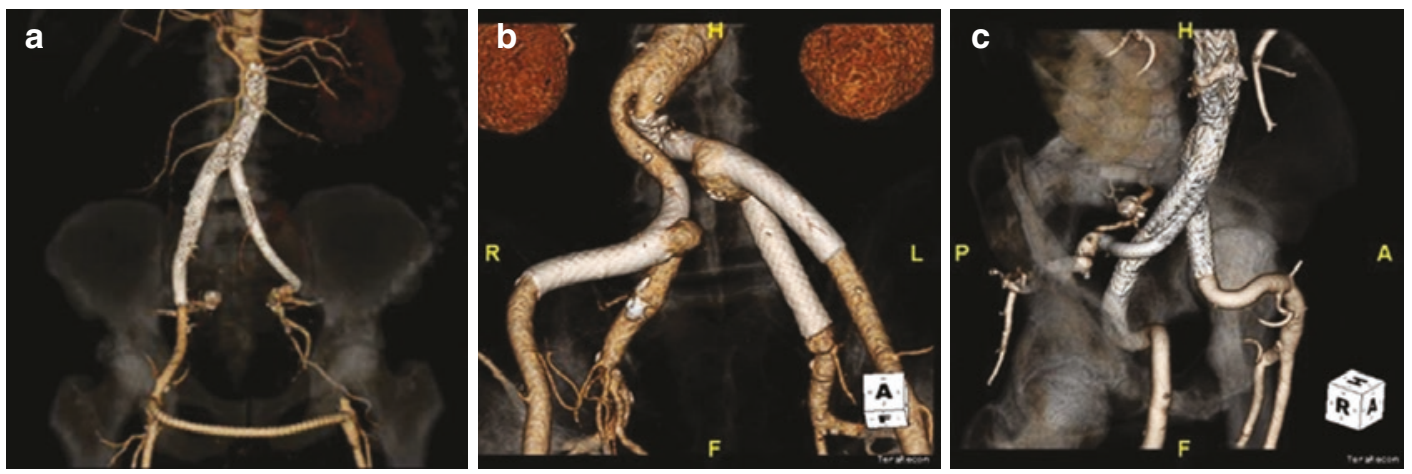

Fig. 8.5 (a) Complex hybrid treatment of bilateral iliac aneurysm with EIA occlusion, (b) multilayer stent technique, and (c) sandwich or double-barrel technique 
Fig. $8.6(a, b)$

Hypogastric preservation with a bell-bottom iliac limb
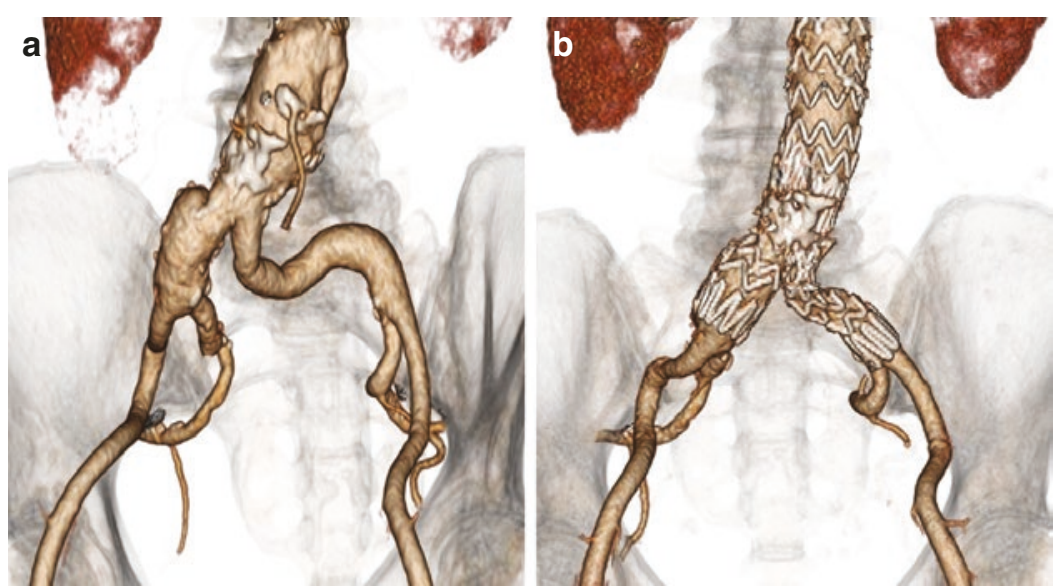

concerning the use of such device in the treatment of visceral and peripheral aneurysms [42, 43], there are still few data concerning its application in the aortoiliac vessels [44], and its effectiveness has still to be determined.

The chimney technique was initially used as a bailout to restore the patency of inadvertently covered vessels (usually renal arteries) during aortic endografting [45]. It consists in the cannulation of target collateral vessels, originating from the covered part of the aorta, parallel to the released graft and stent placement (usually covered or bare metal balloonexpandable stents) to restore antegrade blood flow. The use of this technique has been recently proposed in patients with complex aneurysms, involving visceral and iliac vessels (Fig. 8.5c) [46, 47]. However, with the introduction and technical improvements of fenestrated and branched endografts, the role of the chimney technique remains limited to the treatment of unexpected intraoperative complications or in urgent setting, as custom-made endografts are associated with a not negligible crafting time and off-the-shelf devices may not be available.

When the stents used to preserve flow in the collaterals are released proximally inside an iliac limb or a tube graft, it is called "sandwich technique" (or double-barrel technique) [48, 49]. It has been proposed in patients with extensive thoracoabdominal or aortoiliac aneurysms to achieve proximal seal using a graft as a safe landing zone when a native one is not present. The early- and midterm results are promising; however, the technique is complex and technically demanding, often requiring a brachial access to engage HAs and is often associated with the early phase of the follow-up with the presence of the so-called small-gutter endoleaks. These are technique-specific type III endoleaks related to the mutual disposition of graft materials and generally disappear after a period up to 6 months postoperatively and may require a reintervention (filling the space between the grafts with glue or coils) if this does not happen.

The most widespread technique to treat iliac aneurysms and to prevent early and late complications related to the coverage or embolization of the HA is the iliac branch device $(I B D)$. This technology was born by adapting a small bifurcated body of a standard endograft to fit in a CIA, using a covered stent as bridge between the contralateral gate and a suitable distal neck inside the main trunk of the HA to preserve blood flow to the pelvis while providing a safer distal landing zone in the EIA (Fig. 8.7) $[37,50]$. Iliac branch devices (IBDs) are one of the most popular options when anatomically feasible [3] (Fig. 8.8). Instructions for use (IFU) for IBDs recommend complete aortic repair with a proximal bifurcated aortic endograft. However, in the case of an isolated common iliac artery aneurysm, a stand-alone IBD implant has been proposed when an adequate landing zone is found 

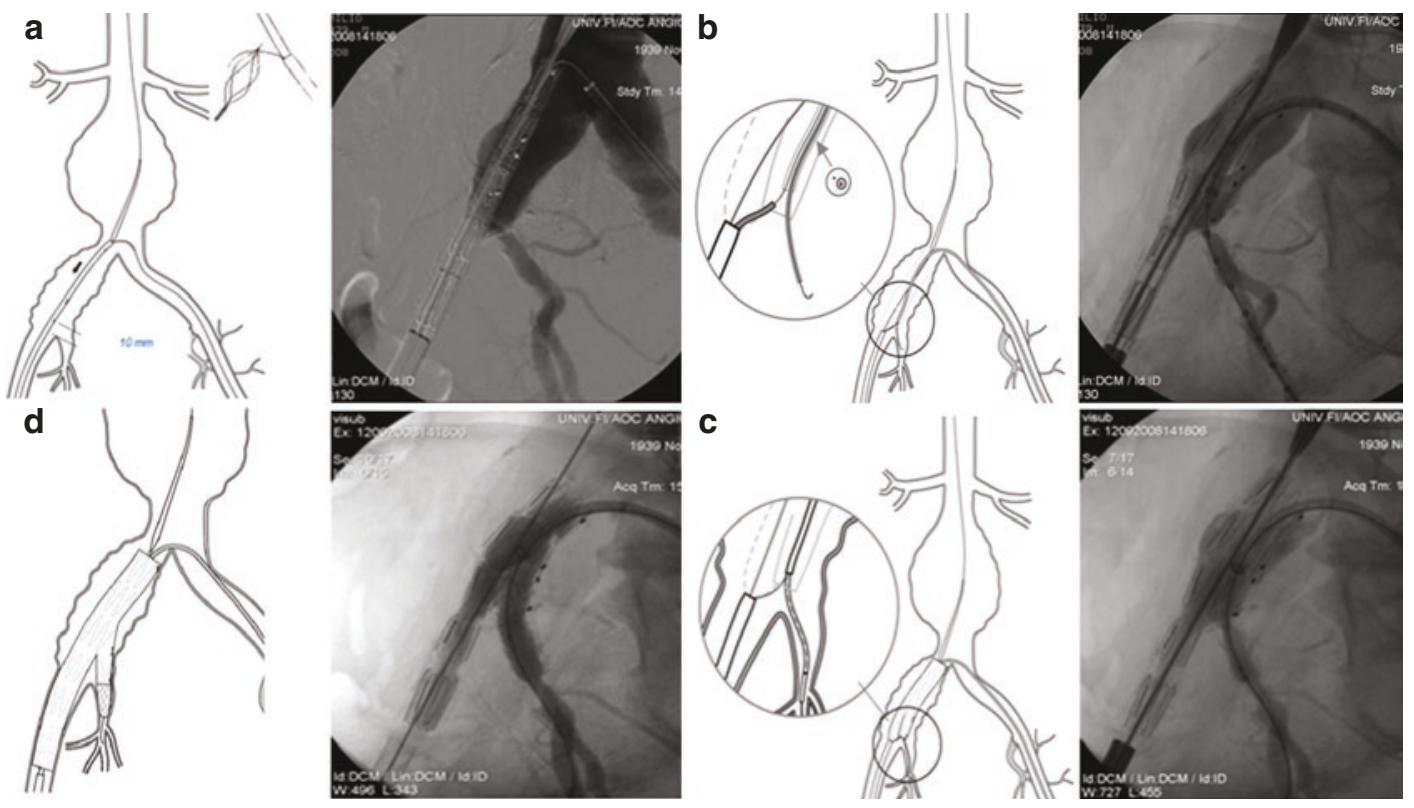

Fig. 8.7 (a-d) Steps of IBD deployment
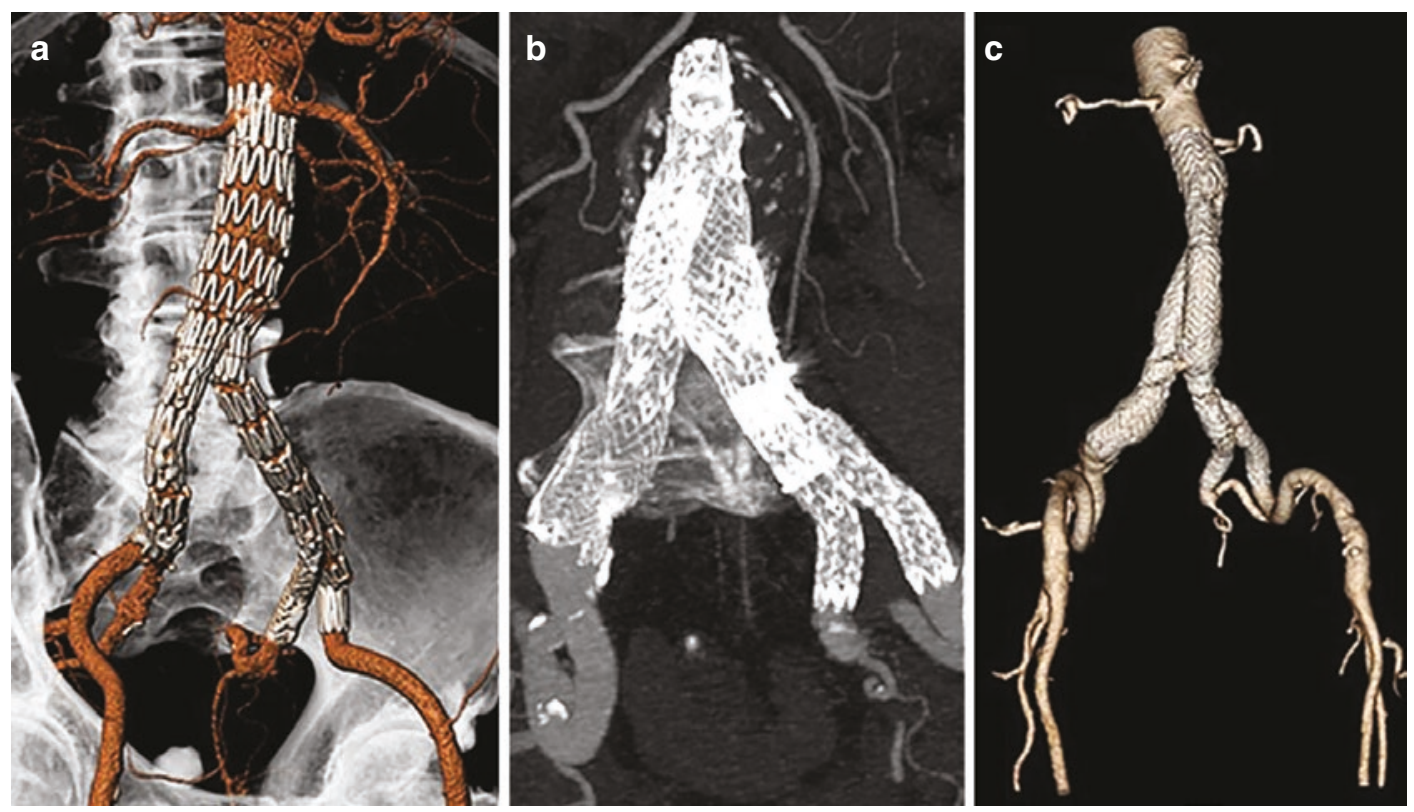

Fig. 8.8 (a) Endovascular exclusion with ZBIS IBD and (b, c) IBE device

in the proximal CIA (Figs. 8.9 and 8.10). The main limitation is related to several anatomical criteria needed to assess the feasibility: a length of CIA $>50 \mathrm{~mm}$, a diameter of the iliac bifurcation $>16 \mathrm{~mm}$, the presence of iliac and femoral access adequate to the dimensions of the devices, a landing zone on the EIA of $20 \mathrm{~mm}$ length with a diameter between 8 and $11 \mathrm{~mm}$, and a landing zone on the HA of $10 \mathrm{~mm}$ length with a diameter adequate for an optimal sealing [51]. Among 

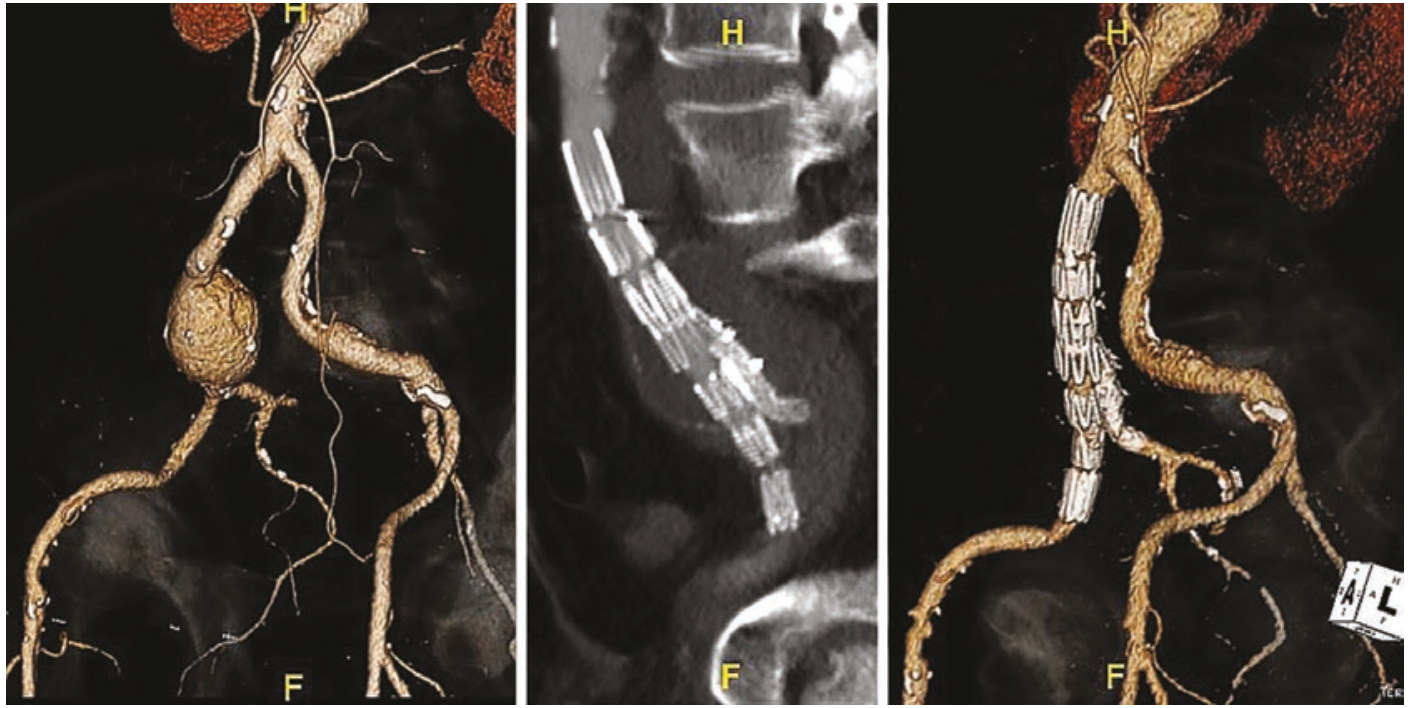

Fig. 8.9 Endovascular exclusion of an IIA with ZBIS IBD

Fig. 8.10 (a) Isolated hypogastric aneurysm and (b) ZBIS IBD landing into distal main trunk
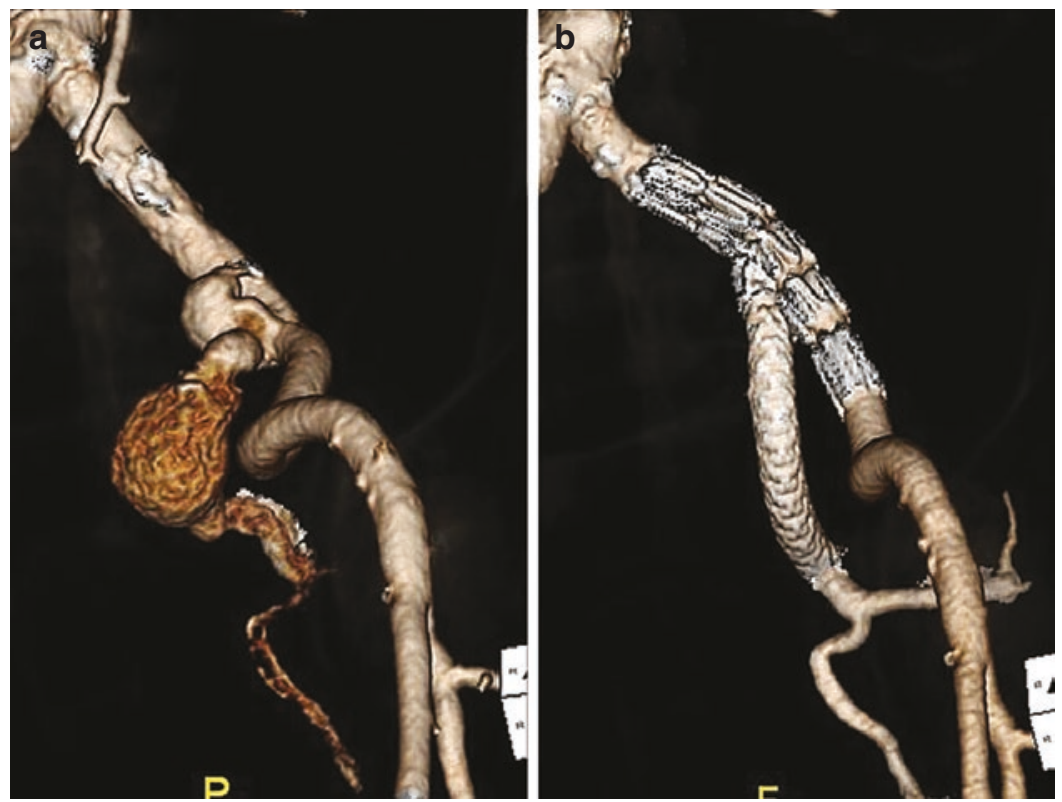

contraindications, severe tortuosity and calcifications of the iliac axis, severe kinking of the iliac bifurcation, and an extensive aneurysmal involvement of the HA are the most commonly encountered (Fig. 8.11). For this reason, a proper case selection along with an accurate planning is mandatory, and a detailed preoperative imaging is crucial. It can usually be obtained with a spiral
angio-CT scan with $1 \mathrm{~mm}$ axial slices and highresolution multiplane reconstructions. Fortunately, the fast evolution of materials has brought about new generation devices that are able to treat even complex anatomies with severe angulations in the origin of the EIA and HA and to reduce potential risk of kinking, dislocation, and migration. Good experience in both aortic 
Fig. 8.11 (a) Wide hypogastric takeoff angle and (b) tight iliac bifurcation
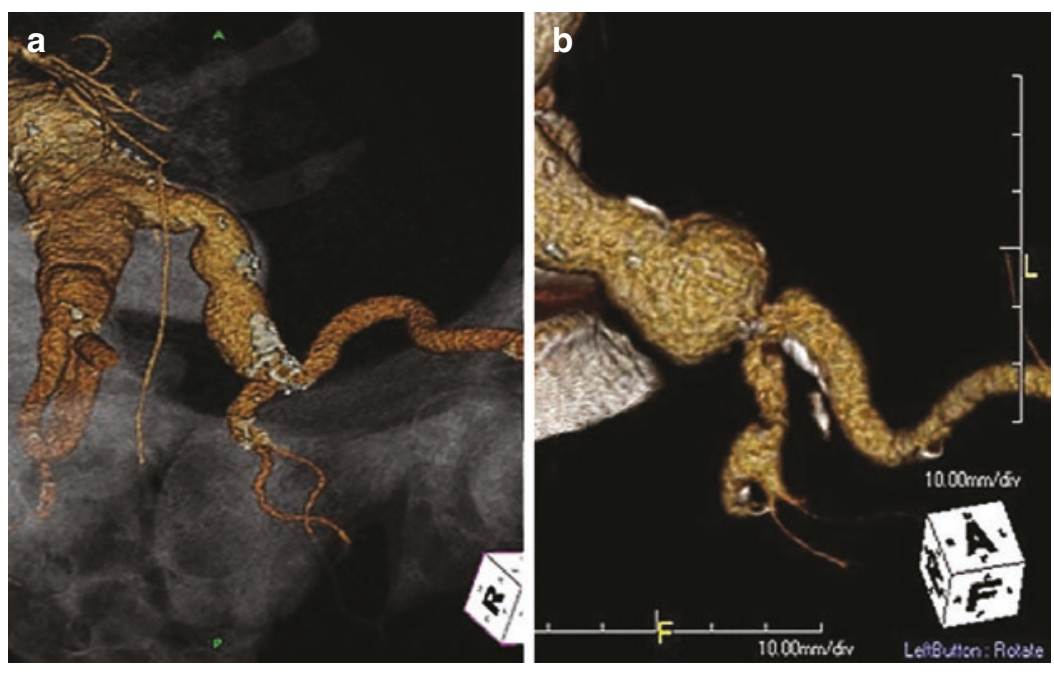

and visceral endovascular interventions together with sufficient advanced endovascular techniques is required to perform an IBD treatment; thus, an adequate learning curve due to the relative complexity of the procedure must be considered.

Several studies have demonstrated that the use of IBD is associated with a reduction in the risk of developing buttock claudication and pelvic ischemia in comparison with alternative techniques [37, 52-54]. Furthermore, a recent retrospective study evaluating the long-term results of IBDs in a series of 575 patients and 650 implants performed in 6 high-volume European centers reported satisfying rates of estimated 8-year freedom from IBD and target HA occlusion (87\% and $95.1 \%$, respectively) and freedom from reintervention due to occlusion and/or type I endoleak (85.7\%) [55]. These results (especially in the mid and long term) allowed the IBD to be considered as the firstchoice treatment in young patients with active lifestyles, who may suffer from a significant impact of ischemic pelvic complications in their everyday activities. Technical success rates approach $97 \%$, and the risk of long-term graftrelated complications is negligible [52]. Moreover, its use is suggested in patients with previous or concomitant treatment of multilevel complex aortic disease (Fig. 8.12) that can benefit from hypogastric flow preservation in terms of prevention of spinal cord ischemia.

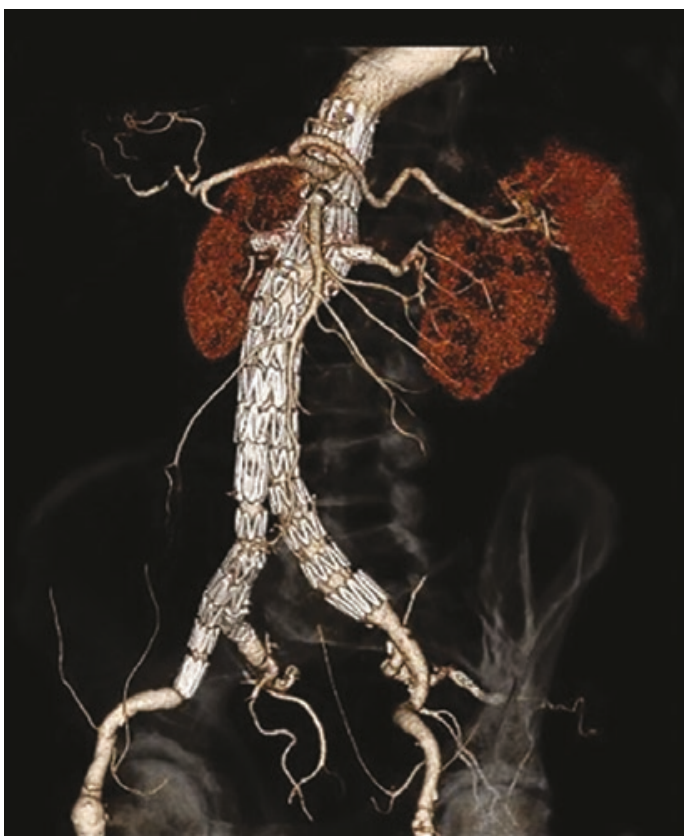

Fig. 8.12 Hypogastric preservation during complex aortic procedures

Recently, the endovascular aneurysm sealing (EVAS), proposed as an alternative method to EVAR for AAA treatment, has been extended also to the visceral and iliac segments. This technique consists of a balloon-expandable stent graft with surrounding polyurethane endobag. Once the stent grafts are deployed, a polymer solution is injected into the endobags to fill the blood 
lumen outside the stents, thereby sealing the aneurysm. These stents have been used also in a "chimney technique" fashion in combination with covered or bare metal stents inside visceral or iliac arteries achieving sealing of the aneurysmal sacs with the bags and leaving blood flow to collaterals. Early results (usually with a mean follow-up less than 12 months) in the literature are also promising in patients with IAAs, but long-term studies are mandatory to assess efficacy and possible complications [56].

\section{References}

1. McCready RA, Pairolero PC, Gilmore JC, et al. Isolated iliac artery aneurysms. Surgery. 1983;93:688-93.

2. Richardson JW, Greenfield LJ. Natural history and management of iliac aneurysms. J Vasc Surg. 1988;8:165-71.

3. Sandhu RS, Pipinos II. Isolated iliac artery aneurysm. Semin Vasc Surg. 2005;18:209-15.

4. Brunkwall J, Hauksson H, Bengtsson H, et al. Solitary aneurysms of the iliac arterial system: an estimate of their frequency of occurrence. J Vasc Surg. 1989;10:381-4.

5. Williams SK, Earnshaw JJ. Intervention criteria for iliac aneurysms. In: Greenhalgh RM, editor. Vascular and endovascular controversies update. London: BIBA; 2012.

6. Giordano J. Embryology of the vascular system. Philadelphia: W. B. Saunders; 2000.

7. Santilli SM, Wernsing SE, Lee ES. Expansion rates and outcomes for iliac artery aneurysms. J Vasc Surg. 2000;31:114-21.

8. Rutherford RB. Management of abdominal aortic aneurysms: which risk factors play a role in decisionmaking? Semin Vasc Surg. 2008;21:124-31.

9. Chaikof EL, Brewster DC, Dalman RL, et al. The care of patients with an abdominal aortic aneurysm: the Society for Vascular Surgery practice guidelines. J Vasc Surg. 2009;50(4 Suppl):2-49.

10. Pratesi C, Alberti V, Apostolou D, et al. Patologia aneurismatica dell'aorta infrarenale, aneurismi viscerali ed aneurismi periferici. Ital J Vasc Endovasc Surg. 2016;23(Suppl 1):1-54.

11. Williams SK, Campbell WB, Earnshaw JJ. Survey of management of common iliac artery aneurysms by members of the Vascular Society of Great Britain and Ireland. Ann R Coll Surg Engl. 2014;96:116-20.

12. Antignani PL, Benedetti-Valentini F, Aluigi L, et al. Diagnosis of vascular diseases. Ultrasound investigations - guidelines of the Italian Society for Vascular Investigation. Int Angiol. 2012;31(5 Suppl 1):1-7.
13. Wilmink AB, Forshaw M, Quick CR, et al. Accuracy of serial screening for abdominal aortic aneurysms by ultrasound. J Med Screen. 2002;9:125-7.

14. Golledge J, Powell JT. Medical management of abdominal aortic aneurysm. Eur J Vasc Endovasc Surg. 2007;34:267-73.

15. Schouten O, van Laanen JH, Boersma E, et al. Statins are associated with a reduced infrarenal abdominal aortic aneurysm growth. Eur J Vasc Endovasc Surg. 2006;32:21-6.

16. Buckley CJ, Buckley SD. Technical tips for endovascular repair of common iliac artery aneurysms. Semin Vasc Surg. 2008;21:31-4.

17. Thompson SG, Brown LC, Sweeting MJ, et al. Systematic review and meta-analysis of the growth and rupture rates of small abdominal aortic aneurysms: implications for surveillance intervals and their cost-effectiveness. Health Technol Assess. 2013;17:1-118.

18. Krupski W. Iliac artery aneurysms. Philadelphia: W. B. Saunders; 2001.

19. Katsargyris A, Oikonomou K, Klonaris C, et al. Common iliac and hypogastric aneurysms: open and endovascular repair. J Cardiovase Surg. 2015;56:249-55.

20. Muehling BM, Ortlieb L, Oberhuber A, et al. Fast track management reduces the systemic inflammatory response and organ failure following elective infrarenal aortic aneurysm repair. Interact Cardiovasc Thorac Surg. 2011;12:784-8.

21. Bacharach JM, Slovut DP. State of the art: management of iliac artery aneurysmal disease. Catheter Cardiovasc Interv. 2008;71:708-14.

22. Levack MM, Bavaria JE, Gorman RC, et al. Rapid aortic arch debranching using the Gore hybrid vascular graft. Ann Thorac Surg. 2013;95(6):163-5.

23. Lachat M, Mayer D, Criado FJ, et al. New technique to facilitate renal revascularization with use of telescoping self-expanding stent grafts: VORTEC. Vascular. 2008;16(2):69-72.

24. Chiesa R, Kahlberg A, Mascia D, et al. Use of a novel hybrid vascular graft for sutureless revascularization of the renal arteries during open thoracoabdominal aortic aneurysm repair. J Vasc Surg. 2014;60(3):622-30.

25. Tyagi S, Pineda D, Zheng H, et al. A novel method for the treatment of bilateral hypogastric aneurysms using hybrid polytetrafluoroethylene graft. Vasc Endovasc Surg. 2017;51(4):199-202.

26. Buck DB, Bensley RP, Darling J, et al. The effect of endovascular treatment on isolated iliac artery aneurysm treatment and mortality. J Vasc Surg. 2015;62:331-5.

27. Hallett JW Jr, Marshall DM, Petterson TM, et al. Graft-related complications after abdominal aortic aneurysm repair: reassurance from a 36-year population-based experience. J Vasc Surg. 1997;25:277-84.

28. Kasirajan V, Hertzer NR, Beven EG, et al. Management of isolated common iliac artery aneurysms. Cardiovasc Surg. 1998;6:171-7. 
29. Dorigo W, Pulli R, Troisi N, et al. The treatment of isolated iliac artery aneurysm in patients with non-aneurysmal aorta. Eur J Vasc Endovasc Surg. 2008;35:585-9.

30. Troisi N, Dorigo W, Marek J, et al. Treatment for isolated iliac aneurysms. In: Setacci C, Gasparini D, Reimers B, Cremonesi A, Rossi P, editors. Aortic surgery: new developments and perspectives. Turin: Edizioni Minerva Medica; 2009. p. 429-3.

31. Parodi JC, Palmaz JC, Barone HD. Transfemoral intraluminal graft implantation for abdominal aortic aneurysms. Ann Vasc Surg. 1991;5:491-9.

32. Greenhalgh RM, Powell JT. Endovascular repair of abdominal aortic aneurysms. $N$ Engl $\mathrm{J}$ Med. 2008;398:454-501.

33. Rayt HS, Bown MJ, Lambert KV, et al. Buttock claudication and erectile dysfunction after internal iliac artery embolization in patients prior to endovascular aortic aneurysm repair. Cardiovasc Intervent Radiol. 2008;31:728-34.

34. Bratby MJ, Munneke GM, Belli AM, et al. How safe is bilateral internal iliac artery embolization prior to EVAR? Cardiovasc Interv Radiol. 2008;31:246-53.

35. Vandy F, Criado E, Upchurch GR Jr, et al. Transluminal hypogastric artery occlusion with an Amplatzer vascular plug during endovascular aortic aneurysm repair. J Vasc Surg. 2008;45:1121-4.

36. Wyers MC, Schermerhorn ML, Fillinger MF, et al. Internal iliac occlusion without coil embolization during endovascular abdominal aortic aneurism repair. J Vasc Surg. 2002;36:1138-45.

37. Verzini F, Parlani G, Romano L, et al. Endovascular treatment of iliac aneurysm: concurrent comparison of side branch endograft versus hypogastric exclusion. J Vasc Surg. 2009;49:1154-61.

38. Yano OJ, Morrissey N, Eisen L, et al. Intentional internal iliac artery occlusion to facilitate endovascular repair of aortoliac aneurysms. J Vasc Surg. 2001;34:204-11.

39. Pratesi G, Pulli R, Fargion A, et al. Alternative hybrid reconstruction for bilateral common and internal iliac artery aneurysms associated with external iliac artery occlusion. J Endovasc Ther. 2009;16:638-41.

40. Karch LA, Hodgson KJ, Mattos MA, et al. Management of ectatic, nonaneurysmal iliac arteries during endoluminal aortic aneurysm repair. Management of ectatic, nonaneurysmal iliac arteries during endoluminal aortic aneurysm repair. J Vasc Surg. 2001;33:33-8.

41. Torsello G, Schönefeld E, Osada N, et al. Endovascular treatment of common iliac artery aneurysms using the bell-bottom technique: long-term results. J Endovasc Ther. 2010;17:504-9.

42. Ruffino M, Rabbia C, Italian Cardiatis Registry Investigators Group. Endovascular treatment of visceral artery aneurysms with Cardiatis multilayer flow modulator: preliminary results at six-month followup. J Cardiovasc Surg. 2011;52:311-21.
43. Sfyroeras GS, Dalainas I, Giannakopoulos TG, et al. Flow-diverting stents for the treatment of arterial aneurysms. J Vasc Surg. 2012;56:839-46.

44. Natrella M, Castagnola M, Navarretta F, et al. Treatment of juxtarenal aortic aneurysm with the Multilayer stent. J Endovasc Ther. 2012;19:121-4.

45. Greenberg RK, Clair D, Srivastava S, et al. Should patients with challenging anatomy be offered endovascular aneurysm repair? J Vasc Surg. 2003;38: 990-6.

46. Moulakakis KG, Mylonas SN, Avgerinos E, et al. The chimney graft technique for preserving visceral vessels during endovascular treatment of aortic pathologies. J Vasc Surg. 2012;55:1497-503.

47. Mosquera Arochena N, Rodríguez Feijoo G, Carballo Fernandez C, et al. Use of modified sandwich-graft technique to preserve hypogastric artery in EVAR treatment of complex aortic aneurysm anatomy. J Cardiovasc Surg. 2011;52:643-9.

48. Lobato AC, Camacho-Lobato L. The sandwich technique to treat complex aortoiliac or isolated iliac aneurysms: results of midterm follow-up. J Vasc Surg. 2013;57:26-34.

49. Lim CS, Naji Y, Hussain ST, et al. Modified sandwichgraft technique employing Aorfix and Viabahn stent-grafts to preserve hypogastric flow in cases of complex aortoiliac and isolated common iliac artery aneurysms including the internal iliac artery ostium. Eur J Vasc Endovasc Surg. 2016;51:364-70.

50. Fernández-Alonso L, Fernández-Alonso S, Grijalba FU, et al. Endovascular treatment of abdominal aortic aneurysms involving iliac bifurcation: role of iliac branch graft device in prevention of buttock claudication. Ann Vasc Surg. 2013;27:851-5.

51. Delay C, Deglise S, Lejay A, et al. Zenith bifurcated iliac side branch device: mid-term results and assessment of risk factors for intraoperative thrombosis. Ann Vasc Surg. 2017;41:141-50.

52. Loth AG, Rouhani G, Gafoor SA, et al. Treatment of iliac artery bifurcation aneurysms with the secondgeneration straight iliac bifurcated device. J Vasc Surg. 2015;62:1168-75.

53. Pratesi G, Fargion A, Pulli R, et al. Endovascular treatment of aorto-iliac aneurysms: four-year results of iliac branch endograft. Eur J Vasc Endovasc Surg. 2013;45:607-9.

54. Simonte G, Parlani G, Farchioti L, et al. Lesson learned with the use of iliac branch devices: single centre 10 year experience in 157 consecutive procedures. Eur J Vasc Endovasc Surg. 2017;54(1):95-103.

55. Donas KP, Inchingolo M, Cao P, et al. Secondary procedures following iliac branch device treatment of aneurysms involving the iliac bifurcation: the pELVIS registry. J Endovasc Ther. 2017;24(3):405-10.

56. Youssef M, Nurzai Z, Zerwes S, et al. Initial experience in the treatment of extensive iliac artery aneurysms with the Nellix aneurysm sealing system. J Endovasc Ther. 2016;23:290-6. 